\title{
Modeling and Simulation of Nano and Multiscale Composites
}

\author{
B. Ramgopal Reddy ${ }^{1 *}$ and K. Ramji ${ }^{2}$ \\ ${ }^{1 *}$ R.V.R \& J.C. College of Engineering, Guntur - 522 019, Andhra Pradesh, India \\ ${ }^{2}$ College of Engineering, Andhra University, Visakhapatnam - 530003 , India \\ 1*brgreddy73@gmail.com, ${ }^{2}$ ramjidme@gmail.com
}

\begin{abstract}
Carbon nanotubes (CNTs) are being used extensively as reinforcing materials in polymer matrix composites because of their high strength, stiffness and resilience, as well as superior mechanical, electrical and thermal properties. Incorporating CNTs in polymer matrix composites can potentially enhance the strength and stiffness of composites significantly. In this paper, the effective elastic properties of nanocomposites (CNTs/Epoxy) at different volume fractions of CNTs and multiscale composites (Glass/CNTs/Epoxy) at 5\% volume fraction of CNTs are evaluated using finite element method (FEM). 3-D finite element models using square representative volume element $(R V E)$ incorporating necessary boundary conditions are developed. For validity the obtained results are compared with that of classical theories of equivalent material properties. Good agreement between them has been observed. Further the effect of CNTintegration in fiber-reinforced composites (three-phase) is also studied.
\end{abstract}

Keywords: Carbon nanotubes, Nanocomposites, Multiscale composites, Finite element method, Representative Volume Element, Effective Elastic Properties

\section{Introduction}

Carbon nanotubes (CNTs), discovered first by Sumio lijima [1], have attracted researcher's great attention due to their exceptional mechanical, chemical, thermal, electrical and even biological properties. Computational approach based on the computer modeling and simulations can play a significant role in the development of CNT based composites. Eventhough experimental based research can ideally be used to determine the elastic properties of nanocomposites and multiscale composites, the experimental synthesis and characterization require the use of sophisticated processing methods and testing equipment, which are expensive. On the other hand, computational modeling of nanocomposites and multiscale composites can be very effective and easily attainable. With the ever increasing power of the computer hardware and simulation software, many of the simulation tasks can now be done on a computer.

The main issues or difficulties in simulations are the proper selection of mathematical models for the problems considered. Meaningful computer simulations are very much dependent on the exactness of the mathematical models for the materials under investigation. For the case of continuum mechanics models in the study of mechanical properties of various materials, the most commonly used numerical method is the finite element method, which has a potential to play an important role in the modeling and simulations of nanocomposites and other materials.

Although CNTs embedded in a matrix have modeled and analyzed extensively and successfully using molecular dynamics [2-5] and continuum mechanics models [6-12]. But, limited research has been done on multiscale composites [13-16]. In the present study the elastic properties of nanocomposites and multiscale composites are evaluated using finite element method and the results are compared with those of classical theories of equivalent mechanical properties. 


\section{Methodology}

For the evaluation of mechanical properties of multiscale composites using theoretical simulation, two step procedure is adopted. Fiber micromechanics model and the Halpin - Tsai model [17] are used for multiscale composites and nanocomposites respectively. In the fiber micromechanics, two sets of material properties are needed: the mechanical properties of the fiber and matrix. Nanocomposites properties obtained by using the Halpin-Tsai model (H-Tsai) are used as matrix properties in the fiber micromechanics to make the system multiscale, i.e., nanoscale of the CNTs and microscale of the fibers. The numerical characterization of nanocomposites and multiscale composites is performed using finite element method.

The following assumptions are made for the nano and multiscale composite simulations

- CNT-matrix bonding is perfect and CNT dispersion in the matrix is uniform

- Each CNT possesses same aspect ratio and mechanical properties

- All CNTs are straight tubes

- No voids in the matrix

- Fiber-matrix bonding is assumed to be perfect.

\section{Modeling of Nanocomposites}

\subsection{Classical Theories of Equivalent Mechanical Properties}

3.11. Rule of Mixtures for Unidirectional Continuous Long CNT: A unidirectional composite may be modeled by assuming reinforcing material to be uniform in diameter and properties, continuous and parallel throughout the composite. The RVE of this composite is shown in figure 1 . It is assumed that perfect bonding exists between the fibers and the matrix.


Figure 1. RVE Model of a Matrix with a Continuous Long CNT [14]

The effective longitudinal modulus $\left(\mathrm{E}_{\mathrm{L}}\right)$ of CNT reinforced composite is calculated by the equation

$$
\mathrm{E}_{\mathrm{L}}=\mathrm{E}_{\mathrm{cnt}} \mathrm{V}_{\mathrm{cnt}}+\mathrm{E}_{\mathrm{m}} \mathrm{V}_{\mathrm{m}}
$$

Where $E_{\mathrm{cnt}}$ and $\mathrm{E}_{\mathrm{m}}$ are respectively the Young's modulus of CNT and matrix, $\mathrm{V}_{\mathrm{cnt}}$ and $\mathrm{V}_{\mathrm{m}}$ are the volume fractions of CNT and matrix respectively.

Poisson's ratio $v_{\mathrm{LT}}=v_{\mathrm{cnt}} \mathrm{V}_{\mathrm{cnt}}+v_{\mathrm{m}}\left(1-\mathrm{V}_{\mathrm{cnt}}\right)$

Where $v_{\mathrm{cnt}}$ and $v_{\mathrm{m}}$ are respectively the Poisson's ratio's of CNT and matrix.

3.12. Halpin-Tsai Model for Short CNT Reinforced Composite: When the reinforcing materials are discontinuous short CNTs, aligned in a polymer matrix as shown in Figure 2 , the effective elastic properties can be determined by using Halpin-Tsai model 

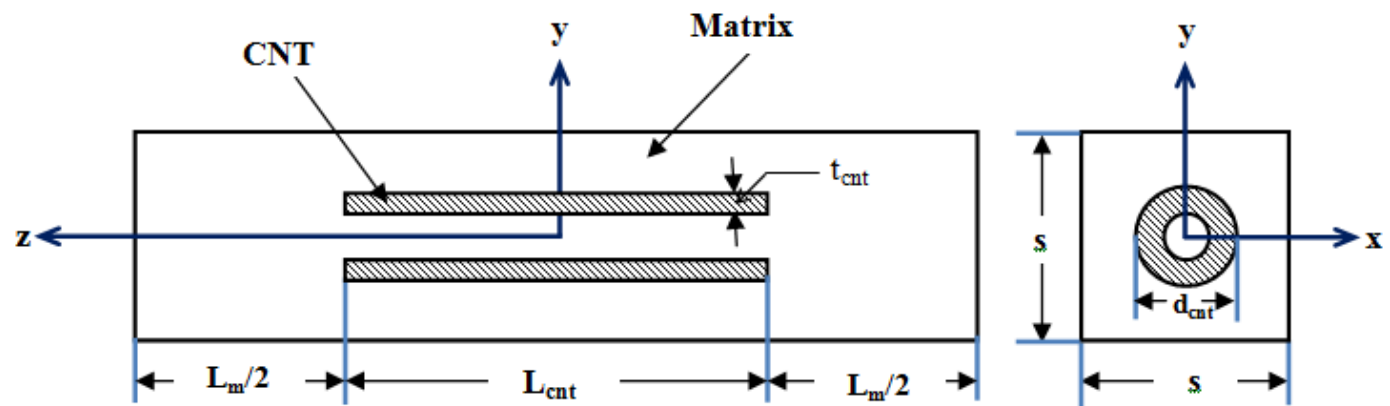

Figure 2. RVE Model of a Matrix with a Discontinuous Short CNT [14]

Halpin-Tsai equations for evaluating the longitudinal modulus $\left(\mathrm{E}_{\mathrm{L}}\right)$ and transverse modulus $\left(\mathrm{E}_{\mathrm{T}}\right)$ are

$\mathrm{E}_{\mathrm{L}}=\left[\frac{1+\left(2 \mathrm{~L}_{\mathrm{cnt}} / \mathrm{d}_{\mathrm{cnt}}\right) \eta_{\mathrm{L}} \mathrm{V}_{\mathrm{cnt}}}{1-\eta_{\mathrm{L}} \mathrm{V}_{\mathrm{cnt}}}\right] \mathrm{E}_{\mathrm{m}}$

Where $\mathrm{L}_{\mathrm{cnt}} / \mathrm{d}_{\mathrm{cnt}}$ is the aspect ratio of CNT and $\eta_{\mathrm{L}}=\frac{\left(\mathrm{E}_{\mathrm{cnt}} / \mathrm{E}_{\mathrm{m}}\right)-1}{\left(\mathrm{E}_{\mathrm{cnt}} / \mathrm{E}_{\mathrm{m}}\right)+2\left(\mathrm{~L}_{\mathrm{cnt}} / \mathrm{d}_{\mathrm{cnt}}\right)}$

$\mathrm{E}_{\mathrm{T}}=\left[\frac{1+2 \eta_{\mathrm{T}} \mathrm{V}_{\mathrm{cnt}}}{1-\eta_{\mathrm{T}} \mathrm{V}_{\mathrm{cnt}}}\right] \mathrm{E}_{\mathrm{m}}$

Where $\eta_{\mathrm{T}}=\frac{\left(\mathrm{E}_{\mathrm{cnt}} / \mathrm{E}_{\mathrm{m}}\right)-1}{\left(\mathrm{E}_{\mathrm{cnt}} / \mathrm{E}_{\mathrm{m}}\right)+2}$

When the reinforcing materials are discontinuous short CNTs, randomly oriented in a matrix, Halpin and Tsai proposed the equations to estimate the effective Young's modulus $\left(\mathrm{E}_{\mathrm{em}}\right)$, Shear modulus $\left(\mathrm{G}_{\mathrm{em}}\right)$ and Poisson's ratio $\left(v_{\mathrm{em}}\right)$ of the composite which is treated as an isotropic medium, i.e.

$\left.\begin{array}{rl}\mathrm{E}_{\mathrm{em}} & =\frac{3}{8} \mathrm{E}_{\mathrm{L}}+\frac{5}{8} \mathrm{E}_{\mathrm{T}} \\ \mathrm{G}_{\mathrm{em}} & =\frac{1}{8} \mathrm{E}_{\mathrm{L}}+\frac{1}{4} \mathrm{E}_{\mathrm{T}} \\ \mathrm{v}_{\mathrm{em}} & =\frac{\mathrm{E}_{\mathrm{em}}}{2 \mathrm{G}_{\mathrm{em}}}-1\end{array}\right\}$

\subsection{Homogenization Method}

A composite is usually composed of fibers and matrix with different properties and will demonstrate non-uniform response when even subjected to a uniform loading in a micromechanical view. But, in classical composite theory the composite is modeled as a homogeneous orthotropic medium with definite effective moduli that describe the average material properties of the composite. One of the most powerful tools to speed up the modeling process, both the composite discretization and the computer simulation of composites in real conditions, is the homogenization method.

In the homogenization method, an RVE shown in Figure 3 is chosen by assuming that the reinforcing material is in a periodic arrangement and it is assumed that the average properties of a RVE is equal to the average properties of the particular composite.

The average stresses and strains in an RVE are defined by

$$
\begin{aligned}
& \overline{\sigma_{\mathrm{ij}}}=\frac{1}{\mathrm{~V}} \int_{\mathrm{V}} \sigma_{\mathrm{ij}} \mathrm{dV} \\
& \overline{\varepsilon_{\mathrm{ij}}}=\frac{1}{\mathrm{~V}} \int_{\mathrm{V}} \varepsilon_{\mathrm{ij}} \mathrm{dV}
\end{aligned}
$$


Where the indices $\mathrm{i}$ and $\mathrm{j}$ denote the global three-dimensional coordinate directions

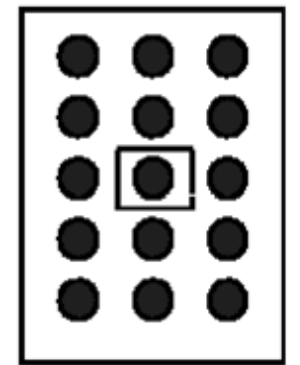

Structure

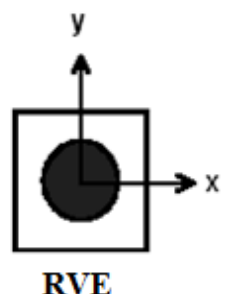

RVE

Figure 3. Periodic Microstructure and an RVE of a Composite

In the present approach, the theory of equivalent homogeneous material proposed by Sun and Vaidya (1996) as a basis to compute the effective moduli of the nanocomposites.

The total strain energy $\mathrm{U}$ stored in the volume $\mathrm{V}$ of the effective medium is given by

$$
\mathrm{U}=\frac{1}{2} \bar{\sigma}_{\mathrm{ij}} \bar{\varepsilon}_{\mathrm{ij}} \mathrm{V}
$$

With the appropriate boundary conditions applied to the RVE model, finite element method can be used to provide the required numerical results by which the effective elastic properties can be calculated by using Hooke's law and strain energy equations as given in equation (9).

$$
\mathrm{E}=\frac{2 \mathrm{U}}{\mathrm{V} \varepsilon^{2}} \text { and } \mathrm{G}=\frac{2 \mathrm{U}}{\mathrm{V} \gamma^{2}}
$$

Where $\mathrm{U}$ is the total energy calculated for the different loading conditions, ' $\varepsilon$ ' is axial strain and ' $\gamma$ ' is the shear strain

\subsection{Numerical Simulation of Nanocomposites}

To evaluate the effective elastic properties of CNT based nanocomposites, an RVE of square cross section for a single-walled carbon nanotube (SWNT) reinforced in a matrix material as shown in Figure 4 is considered. It is assumed that there is a perfect bonding between CNT and matrix.



Figure 4. Square RVE for a SWNT in a Matrix Material (Cutout View) 
Taking advantage of symmetry of the model, only a quarter model of the composite is sufficient enough to simulate and analyze. In the numerical simulation process, SOLID95 element is used to establish a 3D finite element model of the RVE.

The material properties and dimensions taken for the analysis are:

\section{Carbon Nanotube (CNT)}

The type of CNT used in all the cases throughout this research work is the single walled armchair $(9,9)$ type [14]

Outer diameter $\mathrm{d}_{\mathrm{cnt}}=1.22 \mathrm{~nm}$

Effective thickness $\mathrm{t}_{\mathrm{cnt}}=0.34 \mathrm{~nm}$

Length $\mathrm{L}_{\mathrm{cnt}}=50 \mathrm{~nm}$

Young's modulus $\mathrm{E}_{\mathrm{cnt}}=1000 \mathrm{nN} / \mathrm{nm}^{2}$

Poisson's ratio $v_{\mathrm{cnt}}=0.3$

\section{Epoxy Resin Matrix [18]}

Side length of RVEs = variable

Length $\mathrm{L}_{\mathrm{m}}=100 \mathrm{~nm}$

Young's modulus $\mathrm{E}_{\mathrm{m}}=3.5 \mathrm{nN} / \mathrm{nm}^{2}$

Poisson's ratio $v_{\mathrm{m}}=0.3$

The elastic properties of the epoxy matrix and CNT are considered as isotropic.

The effective elastic properties of CNT based polymer composites are evaluated from the total strain energy acquired, which can be obtained by the finite element analysis applying necessary boundary conditions and loading. The results obtained by finite element analysis are given in Table 1.

Table 1. Effective Elastic Properties of the Nanocomposite at Different Volume Fraction of CNTs

\begin{tabular}{|c|c|c|c|c|c|c|c|}
\hline \multirow{2}{*}{$\begin{array}{c}\text { CNT volume } \\
\text { fraction (\%) }\end{array}$} & \multicolumn{4}{|c|}{ Aligned CNT/Epoxy } & \multicolumn{3}{c|}{ Randomly distributed } \\
\cline { 2 - 8 } & $\begin{array}{c}\mathrm{E}_{\mathrm{L}} / \mathrm{E}_{\mathrm{m}} \\
\mathrm{FEM}\end{array}$ & $\begin{array}{c}\mathrm{E}_{\mathrm{L}} / \mathrm{E}_{\mathrm{m}} \\
\text { Analytical }\end{array}$ & $\begin{array}{c}\mathrm{E}_{\mathrm{T}} / \mathrm{E}_{\mathrm{m}} \\
\mathrm{FEM}\end{array}$ & $\begin{array}{c}\mathrm{E}_{\mathrm{T}} / \mathrm{E}_{\mathrm{m}} \\
\text { Analytical }\end{array}$ & $\begin{array}{c}\mathrm{E}_{\mathrm{em}} / \mathrm{E}_{\mathrm{m}} \\
\mathrm{H}-\mathrm{Tsai}\end{array}$ & $\begin{array}{c}\mathrm{G}_{\mathrm{em}} / \mathrm{G}_{\mathrm{m}} \\
\mathrm{H}-\mathrm{Tsai}\end{array}$ & $\begin{array}{c}v_{\mathrm{em}} / v_{\mathrm{m}} \\
\mathrm{H}-\mathrm{Tsai}\end{array}$ \\
\hline 1.0 & 1.583 & 1.632 & 1.029 & 1.064 & 1.237 & 1.183 & 1.196 \\
\hline 2.0 & 1.740 & 1.783 & 1.060 & 1.104 & 1.315 & 1.255 & 1.206 \\
\hline 3.0 & 1.817 & 1.835 & 1.091 & 1.135 & 1.363 & 1.300 & 1.210 \\
\hline 5.0 & 1.868 & 1.901 & 1.123 & 1.176 & 1.402 & 1.336 & 1.213 \\
\hline & 1.902 & 1.937 & 1.157 & 1.209 & 1.436 & 1.370 & 1.216 \\
\hline
\end{tabular}

From the results given in Table 1, it is observed that the longitudinal modulus increases as CNT volume fraction increases. This is evident from the fact that the modulus of CNT is $1 \mathrm{TPa}$, which is about 286 times that of for the matrix and also tensile properties are fiber dominant properties.

It can also be noted that, with the addition of $1 \% \mathrm{CNT}$ in a matrix, the effective modulus in the longitudinal direction of the homogeneous matrix increases by about $59 \%$ and $24 \%$ in case of aligned and randomly oriented CNTs respectively. Effective modulus increases as the percentage of CNT volume increases. In case of aligned and randomly oriented CNT composites, at 5\% CNT volume fraction the increase in axial modulus is 
about $90 \%$ and $44 \%$ respectively and the increase in shear modulus is about $37 \%$ in case of randomly oriented CNT composites.

\section{Modeling and Simulation of Multiscale Composites}

Since all the components of this composite are in quite different scale (glass fiber is in microscale and CNTs are in nanoscale) to form a multi-scale problem, it is complex to construct a suitable RVE model. Instead of modeling this CNTs/Glass fiber/Polymer system directly, combining the polymer and the CNTs into an equivalent homogeneous matrix by the homogenization process and then constructing the RVE model of this homogeneous matrix and glass fiber to perform the subsequent analysis procedure.

The material elastic properties and dimensions taken for the analysis are given below:

Equivalent matrix:

Young's modulus $\mathrm{E}_{\mathrm{em}}=$ Variable

Poisson's ratio $v_{\mathrm{em}}=$ Variable

Length $\mathrm{L}_{\mathrm{em}}=100 \mu \mathrm{m}$

Side length $\mathrm{s}_{\mathrm{em}}=$ Variable

Glass Fiber [18]:

Volume fraction of matrix $\mathrm{V}_{\mathrm{m}}=$ Variable

Young's modulus $\mathrm{E}_{\mathrm{f}}=70 \mathrm{GPa}$

Poisson's ratio $v_{\mathrm{f}}=0.2$

Length of fiber $\mathrm{L}_{\mathrm{f}}=100 \mu \mathrm{m}$

Diameter $\mathrm{d}_{\mathrm{f}}=20 \mu \mathrm{m}$

Volume fraction of fiber $V_{f}=$ Variable

To evaluate the effective elastic properties of multiscale composites, a square RVE for a glass fiber reinforced in a homogeneous matrix material is considered. Due to the symmetry of the model, only quarter model of the multiscale composite as shown in figure 5 is sufficient enough to simulate and analyze.

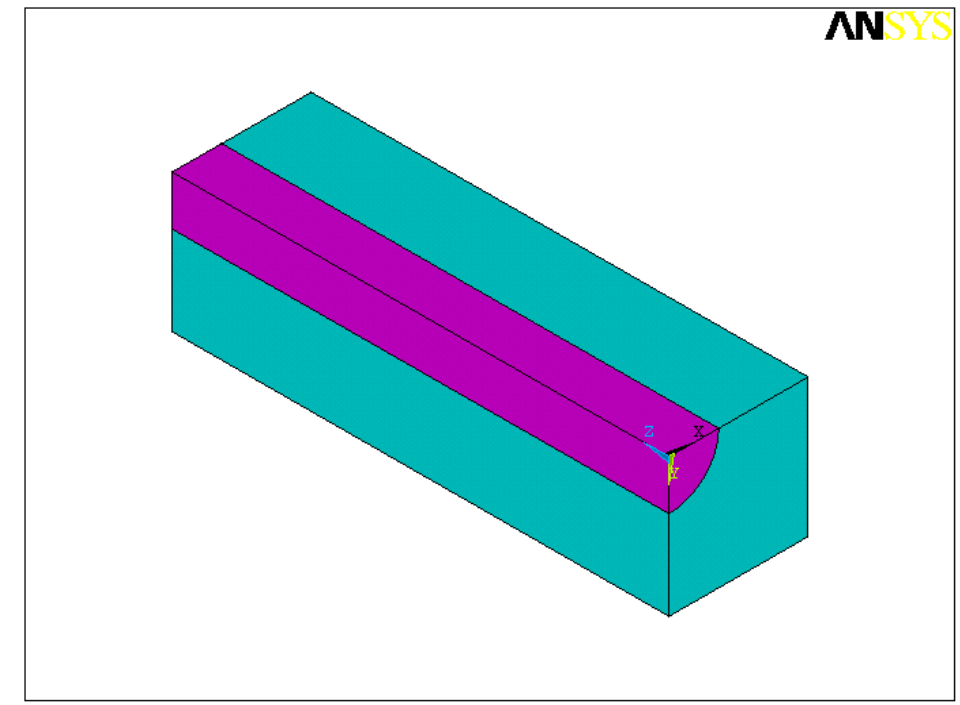

Figure 5. A Quadrant of Square RVE for Multiscale Composite

In the numerical simulation process, SOLID95 element having three degrees of freedom per node: translations along $\mathrm{x}, \mathrm{y}$ and $\mathrm{z}$ directions, is used to establish a 3D finite element model of the RVE. A 3-D quarter model of the RVE using different mesh densities has been developed and the convergence study is carried out in order to determine an appropriate mesh density. The finite element model which is having 74664 
elements and 107000 nodes producing converging results shown in Figure 6 is used for this study.

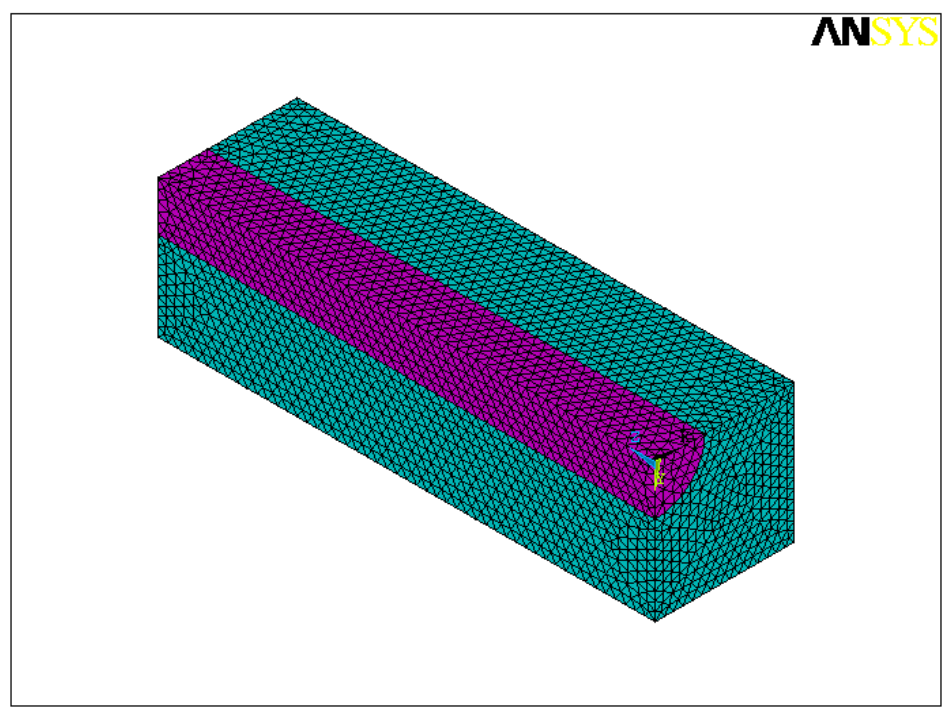

\section{Figure 6. Meshed Model of a Quadrant of Square RVE for a Multiscale Composite}

\section{Results and Discussion}

The effective elastic properties of multiscale composites are evaluated by adding CNTs at $5 \%$ volume fraction into the glass fiber composite (glass fiber volume fraction varying between $10 \%$ and $70 \%$ in steps of $10 \%$ ). The results obtained by finite element simulation are presented in Figures 7-10.

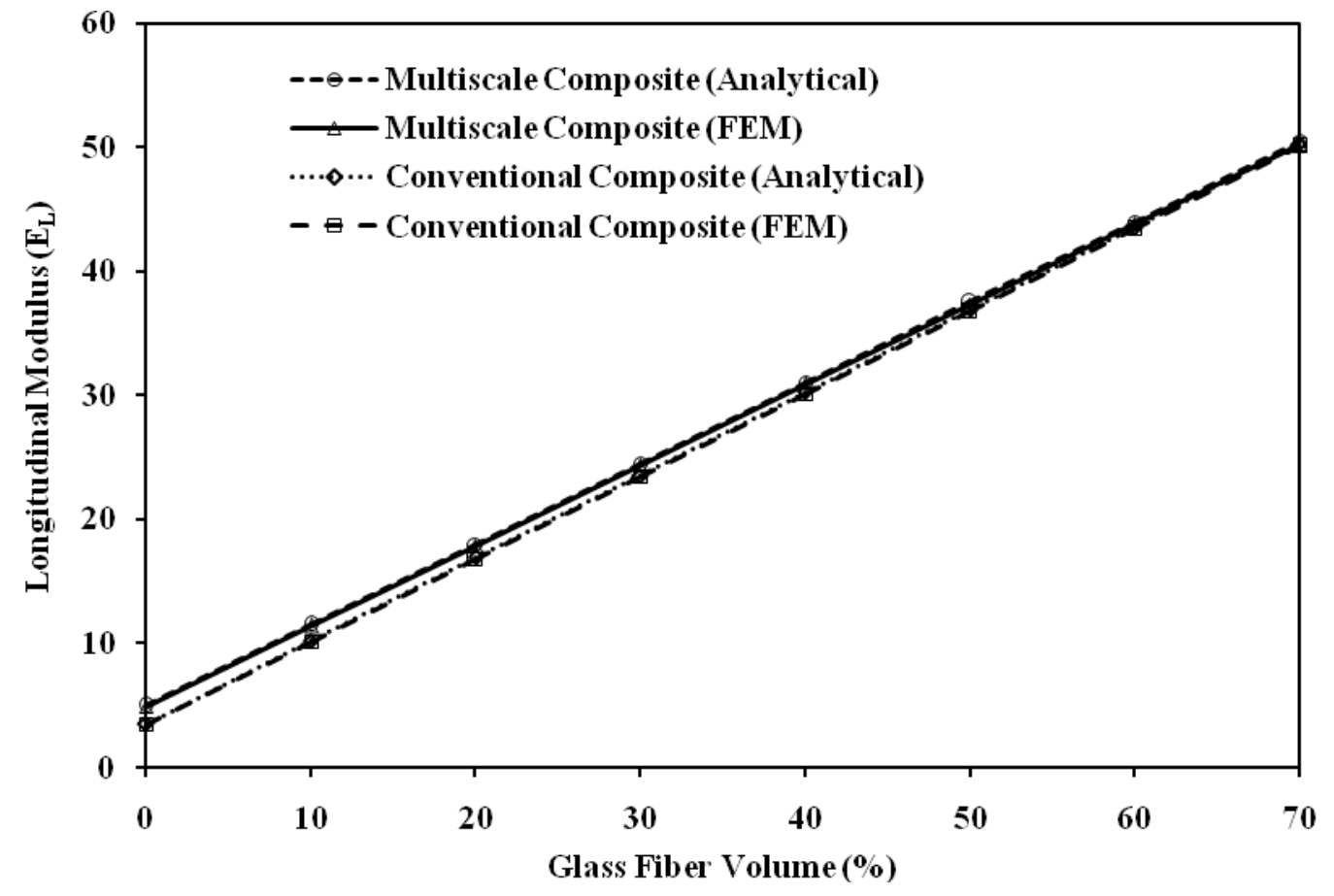

Figure 7. Effect of Glass Fiber Volume Fraction on Longitudinal Modulus at $5 \%$ CNT Volume Fraction 


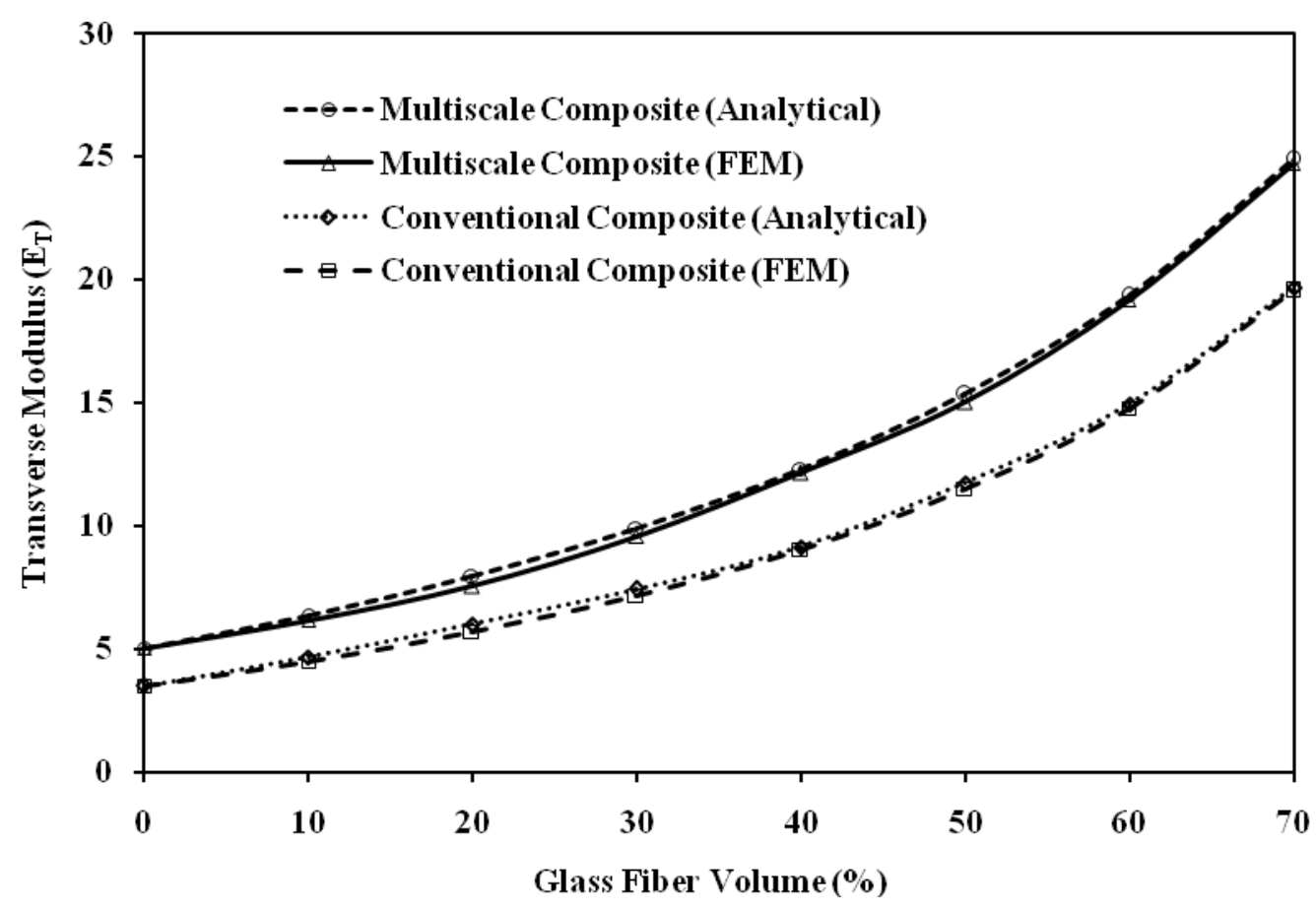

Figure 8. Variation of Transverse Modulus with Glass Fiber Volume Fraction at $5 \%$ CNT Volume Fraction



Figure 9. Effect of Glass Fiber Volume Fraction on Shear Modulus at $5 \%$ CNT Volume Fraction 


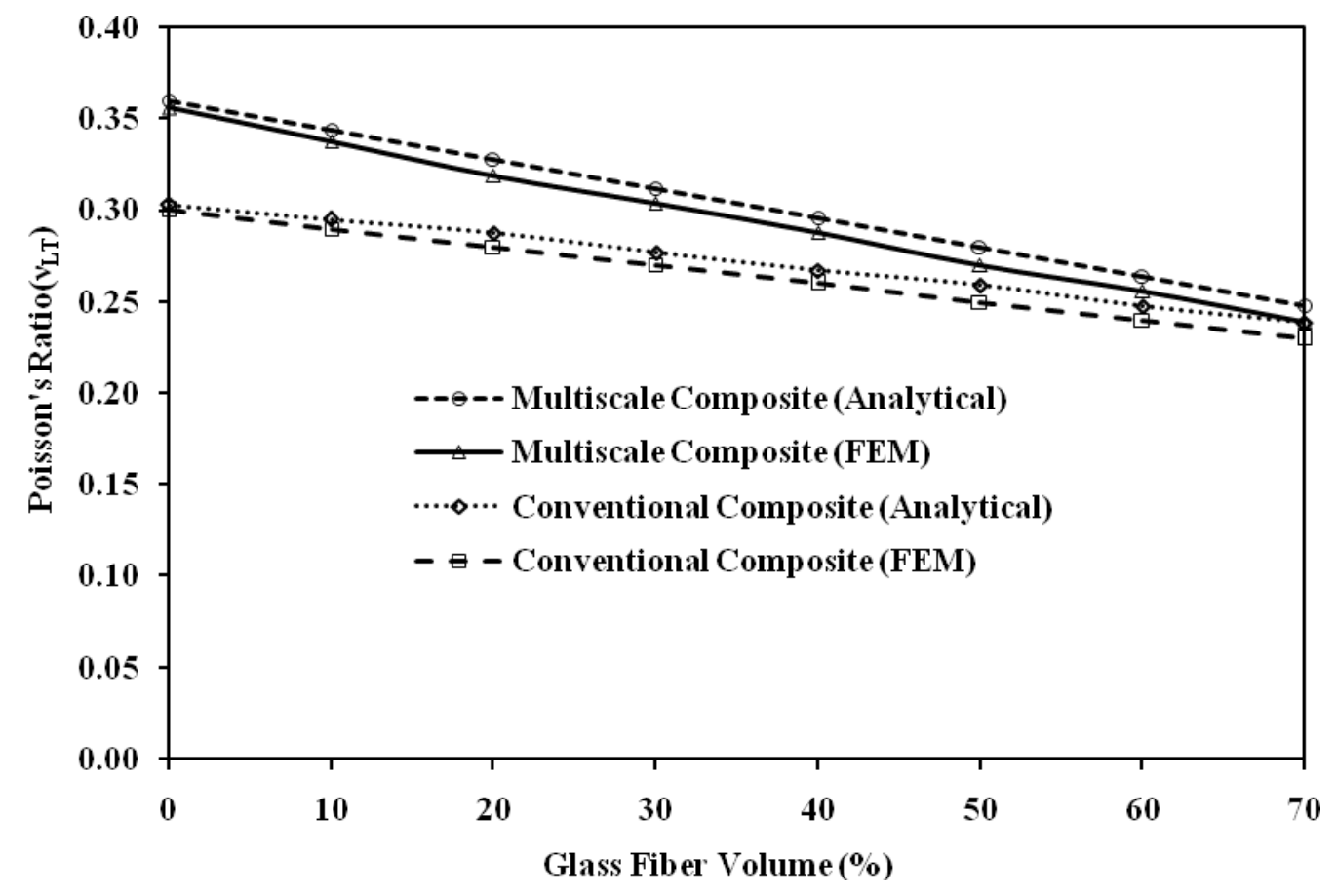

\section{Figure 10. Variation of Poisson's Ratio with Glass Fiber Volume Fraction at $5 \%$ CNT Volume Fraction}

Effective elastic properties such as longitudinal modulus, transverse modulus, shear modulus and Poisson's ratio with respect to glass fiber volume fraction are evaluated for $5 \%$ volume fraction of CNTs. The numerical results are compared with the analytical results and observed that the maximum deviation between these results is within $4 \%$, which indicates that there is a good agreement between the two.

Figure 7 show the variation of effective longitudinal modulus of multiscale composites with respect to fiber volume fraction for different volume fractions of CNTs. From the plot it can be noted that, reinforcing effect of adding CNTs into glass fiber composite, the effective longitudinal modulus increases as the CNT volume fraction increases. It is also observed that at $10 \%$ fiber volume fraction and at 5\% volume fraction of CNTs, the increase in longitudinal modulus is about $13.7 \%$. But the percentage increase in effective longitudinal modulus decreases as the volume fraction of fiber increases. At 5\% CNT volume fraction and $70 \%$ fiber volume fraction the increase in longitudinal modulus is only $1 \%$. In depth study reveals that the reduction in percentage increase in longitudinal modulus is due to less number of CNTs available as the percentage of fiber volume fraction increases.

The variation of effective transverse elastic modulus with fiber volume fraction for $5 \%$ volume fraction of CNTs is shown in figure 8. From the plot it can be noted that there is a significant increase in transverse modulus due to the reinforcement of CNTs into glass fiber composites. It is also observed that at $10 \%$ fiber volume and 5\% CNT volume fractions, the effective transverse modulus of multiscale composite increases by about $42 \%$ than the conventional composite. But the percentage increase in transverse modulus decreases as the volume fraction of fiber increases. In depth observations reveal that, the decrease is due to less number CNTs available as the fiber volume fraction increases. At $5 \%$ CNT volume fraction and $70 \%$ fiber volume fraction the increase in transverse modulus is about $27 \%$.

Figure 9 show the variation of effective shear modulus with fiber volume fraction for CNT volume fraction 5\%. From the plot it can be observed that due to the reinforcement of CNTs in glass fiber composites, there is a significant increase in shear modulus. At 
$10 \%$ fiber volume and 5\% CNT volume fraction, the effective shear modulus of multiscale composite increases by about $43 \%$ than the conventional composite. But the percentage increase in effective modulus decreases as the fiber volume fraction increases and this is due to less number CNTs available as the fiber volume increases. At $70 \%$ fiber volume fraction and 5\% CNT volume fraction the effective transverse modulus increases by about $32 \%$.

The variation of Poisson's ratio with fiber volume fraction for volume fraction of CNTs $5 \%$ is shown in figure 10 . The Poisson's ratio mainly depends on the transverse and longitudinal strains. However longitudinal strain is fiber dominant property, obviously there is a significant decrease in Poisson's ratio by increasing fiber volume fraction.

From the results it can be noted that by adding CNTs, the increase in effective longitudinal modulus of multiscale composites is not as good as in the nanocomposites. This is due to tensile properties of multiscale composites are fiber-dominant properties. Therefore longitudinal elastic modulus depends highly on the fiber volume fraction, not on the CNT volume fraction. However, there is a significant increase in effective transverse modulus as well as effective shear modulus of multiscale composites due to CNTs reinforcement, which shows that the CNTs have best reinforcement effect in the transverse direction.

\section{Conclusions}

In the present work, effective elastic properties of nanocomposites at different volume fraction of CNTs varying from 1 to $5 \%$ and multiscale composites at 5\% volume fraction of CNTs are evaluated. It is observed that low volume fraction (1 to 5\%) of CNTs results in large increase in effective elastic properties of nanocomposites.

The reinforcing effect of adding CNTs into glass fiber composites (multiscale composites), the increase in effective longitudinal elastic modulus is not that much significant. However, the effective transverse elastic modulus and shear modulus have been found increasing significantly.

The results clearly indicate that incorporating CNTs in polymer matrix composites can potentially enhance the mechanical properties of composites significantly.

\section{References}

[1] S. Iijima, "Helical microtubules of graphitic carbon", Nature, vol. 354, (1991), pp. 56-58.

[2] S. J. V. Frankland, V. M. Harik, G. M. Odegard, D. W. Brenner and T. S. Gates, "The stress-strain behavior of polymer-nanotube composites from molecular dynamics simulation", Composites Science and Technology, vol. 63, (2003), pp. 1655-1661.

[3] R. Zhu, E. Pan and A. K. Roy, "Molecular dynamics study of the stress-strain behavior of carbonnanotube reinforced Epon 862 composites", Material Science \& Engineering, vol. 447, (2007), pp. 51-57.

[4] Q. H. Zeng, A. B. Yu and G. Q. Lu, "Multiscale modeling and simulation of polymer nanocomposites", Progress in Polymer Science, vol. 33, (2008), pp.191-269.

[5] B. Arash, Q. Wang and V. K. Varadan, "Mechanical properties of carbon nanotube/polymer composites", Scientific Reports, vol. 4:6479, (2014), pp. 1-8.

[6] C. T. Sun and R. S. Vaidya, "Prediction of composite properties from a representative volume element", Composites Science and Technology, vol. 56, no. 2, (1996), pp.171-179.

[7] K. Sohlberg, B. G. Sumpter, R. E. Tuzun and D. W. Noid, "Continuum methods of mechanics as a simplified approach to structural engineering of nanostructures", Nanotechnology, vol. 9, (1998), pp. 30-36.

[8] Y. J. Liu and X. L. Chen, "Evaluations of the effective material properties of carbon nanotube-based composites using a nanoscale representative volume element", Mechanics of Materials, vol. 35, (2003), pp. 69-81.

[9] G. M. Odegard, S. J. V. Frankland and T. S. Gates, "Effect of nanotube functionalization on the elastic properties of polyethylene nanotube composites", AIAA, vol. 43, no. 8, (2005), pp. 1828-1835.

[10] Y. S. Song and J. R. Youn, "Modeling of effective elastic properties for polymer based carbon nanotube composites", Polymer, vol. 47, (2006), pp. 1741-1748. 
[11] R. C. Batra and A. Sears, "Continuum models of multi-walled carbon nanotubes", Solids and Structures, vol.44, (2007), pp. 7577-7596.

[12] A. K. Gupta and S. P. Harsha, "Analysis of mechanical properties of carbon nanotube reinforced polymer composites using continuum mechanics approach", Procedia Materials Science, vol. 6, (2014), pp. $18-25$.

[13] E. Bekyarova, E. T. Thostenson, A. Yu, K. Kim, J. Gao, J. Tang, H. T. Hahn, T-W. Chou, M. E. Itkis and R. C. Haddon, "Multiscale carbon nanotube-carbon fiber reinforcement for advanced epoxy composites", Langmuir, vol. 23, no. 7, (2007), pp. 3970-3974.

[14] C. W. Fan, C. S. Lin and C. Hwu, "Numerical estimation of the mechanical properties of CNTreinforcing composites", Proceedings of fifth Taiwan-Japan work shop on Mechanical and Aerospace Engineering, Nantou, Taiwan, (2009) October 21-24.

[15] L. Mei, Y. Li, R. Wang, Ch. Wang, Q. Peng and X. He, "Multiscale carbon nanotube-carbon fiber reinforcement for advanced epoxy composites with high interfacial strength", Polymers and Polymer Composites, vol. 19, no. 2-3, (2011), pp. 107-112.

[16] M. Kim, Y. Park, O. I. Okoli and C. Zhang, "Processing, characterization and modeling of carbon nanotube-reinforced multiscale composites", Composites Science and Technology, vol. 69, (2009), pp. 335-342.

[17] J. C. Halpin and J. L. Kardos, "The Halpin-Tsai equations: A review", Polymer Engineering and Science, vol. 16, no. 5, (1976), pp. 344-352.

[18] B. D. Agarwal, L. J. Broutman and K. Chandrashekhara, "Analysis and performance of fiber composites,” John Wiley and sons, Inc., Hoboken, New Jersey, (2006).

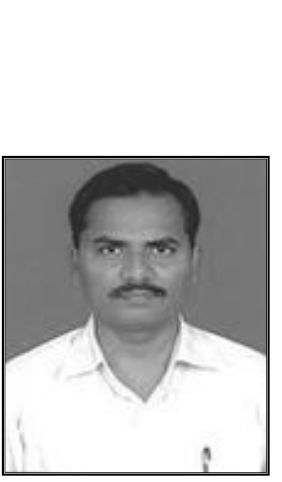

\begin{abstract}
Authors
B. Ramgopal Reddy, He is working as an Associate Professor in the Department of Mechanical Engineering at R.V.R. \& J.C. College of Engineering, Guntur, Andhra Pradesh, India. He obtained his B. Tech. degree in Mechanical Engineering from Nagarjuna University in 1996, M. Tech. degree in 1998 from National Institute of Technology, Warangal. He obtained his Ph.D. from Andhra University, Visakhapatnam in the area of Carbon Nanotube Based Multiscale Composites. He has more than twenty research papers in International/National journals and conferences to his credit. His research areas include Composite Materials, FEM, and Machine design.
\end{abstract}

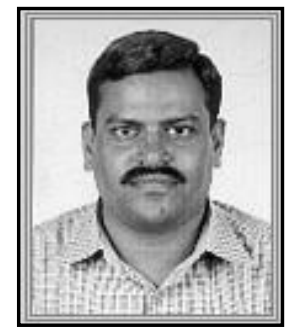

K. Ramji, He obtained his Ph.D. degree from IIT Roorkee. He has published more than 150 technical papers in various national and international journals and conferences and guided 48 students for $\mathrm{M}$. Tech. and 11 Students for Ph.D. Another 10 students are pursuing their Ph.D. work. He is also the recipient of "Best researcher award" of the year 2006 and "Dr. Sarvepalli Radhakrishnan Award for Best Academician" of the year 2007. Currently he is working as Professor in the department of mechanical engineering at A.U. College of Engineering, Andhra University, Visakhapatnam, Andhra Pradesh. His research interests include Vehicle Dynamics, FEM, CAD, Machine Design, Robotics and Nano Technology. 
International Journal of Hybrid Information Technology Vol.9, No.3 (2016) 